\title{
Evaluating the associations between human circadian rhythms and dysregulated genes in liver cancer cells
}

\author{
ANDREA POLO $^{1}$, SAKSHI SINGH ${ }^{2}$, ANNA CRISPO $^{1}$, MARILINA RUSSO $^{3}$, ALDO GIUDICE $^{1}$, \\ MAURIZIO MONTELLA ${ }^{1}$, GIOVANNI COLONNA ${ }^{4}$ and SUSAN COSTANTINI ${ }^{3}$ \\ ${ }^{1}$ Epidemiology Unit, National Cancer Institute 'Foundation G. Pascale', IRCCS; \\ ${ }^{2}$ Doctorate in Computational Biology, Second University of Naples; ${ }^{3}$ Oncology Research Center of \\ Mercogliano, National Cancer Institute 'Foundation G. Pascale', IRCCS; ${ }^{4}$ Medical Informatics Service, \\ University Hospital, University of Campania 'Luigi Vanvitelli', I-80131 Naples, Italy
}

Received March 28, 2017; Accepted August 24, 2017

DOI: $10.3892 / 01.2017 .7109$

\begin{abstract}
Network analysis is a useful approach in cancer biology as it provides information regarding the genes and proteins. In our previous study, a network analysis was performed on dysregulated genes in HepG2 cells, a hepatoblastoma cell line that lacks the viral infection, compared with normal hepatocytes, identifying the presence of 26 HUB genes. The present study aimed to identify whether these previously identified HUB genes participate in the network that controls the human circadian rhythms. The results of the present study demonstrated that 20/26 HUB genes were associated with the metabolic processes that control human circadian rhythms, which supports the hypothesis that a number of cancer types are dependent from circadian cycles. In addition, it was revealed that the CLOCK circadian regulator gene was associated, via cytoskeleton associated protein 5 (CKAP5), with the HUB genes of the HepG2 network, and that CKAP5 was associated with three other circadian genes (casein kinase $1 \varepsilon$, casein kinase $1 \delta$ and histone deacetylase 4) and 10 HepG2 genes ( $\mathrm{SH} 2$ domain containing, ZW10 interacting kinetochore protein, aurora kinase $\mathrm{B}$, cell division cycle 20 , centromere protein $\mathrm{A}$, inner centromere protein, mitotic arrest deficient 2 like 1, baculoviral IAP repeat containing 5, SPC24 NDC80 kinetochore complex component and kinesin family member 2C). Furthermore, the genes that associate the circadian system with liver cancer were demonstrated to encode intrinsically disordered proteins. Finally, the results of the present study identified the microRNAs involved in the network formed by the overlapping of HepG2 and circadian genes.
\end{abstract}

Correspondence to: Dr Susan Costantini, Oncology Research Center of Mercogliano, National Cancer Institute 'Foundation G. Pascale', IRCCS, Mariano Semmola 52, I-80131 Naples, Italy E-mail: s.costantini@istitutotumori.na.it

Key words: network analysis, circadian genes, hepatocellular carcinoma, HUB genes

\section{Introduction}

Circadian rhythms control the $24 \mathrm{~h}$ cycle of specific metabolic functions required by living beings, ensuring an efficient metabolic homeostasis $(1,2)$. In humans, the circadian rhythms are controlled by a master pacemaker situated in the suprachiasmatic nuclei of the hypothalamus, which is synchronized to the photoperiod $(1,2)$. The molecular clock of transcription involves a translational feedback mechanism of genes, including clock circadian regulator (CLOCK), period circadian clock (PER), aryl hydrocarbon receptor nuclear translocator like (BMAL1) and cryptochrome circadian clock (CRY), able to regulate a number of physiological properties, including body temperature, melatonin secretion, hormone secretion, blood pressure and the sleep-wake cycle (3).

It is well-known that disturbances in the circadian rhythm may cause the development of diseases, including major depressive disorder, seasonal affective disorder, schizophrenia, bipolar disorder (4-9), stress, desynchronosis (9), anxiety disorder, diabetes (10), obesity, diseases associated with aging (11), genome instability (12) and cancer $(13,14)$. Prior studies have demonstrated the association between circadian rhythm alterations and the development of breast (15) and prostate cancer (16), B-cell lymphoma (17), non-small cell lung (18), testicular (19) and ovarian cancer (20).

The liver has a central and unique metabolic function in maintaining energy homeostasis via glycolysis and gluconeogenesis associated with fatty acid metabolism (biosynthesis/beta oxidation) (21). Rhythmic fluctuations have been identified in hepatic metabolic functions with a 24-h periodicity (22). Previous studies have demonstrated that liver cancer initiation may be due to alterations in circadian rhythmic genes, including PER3 (23) and CRY genes, and casein kinases (24). Additionally, it has been revealed that the dysregulation of metallothionein-1 (MT-1), MT-2 and metal transcription factor-1 are involved in the alterations to circadian rhythms present in liver cancer (25). Furthermore, the presence of hepatitis viral infection, which is a cause of liver cancer, has been revealed to cause dysregulation of the expression of circadian genes (26). However, it is crucial to underline that liver cancer is additionally caused by chronic 
exposure to toxic chemicals, hepatosteatosis, type 2 diabetes and obesity (27-29). The underlying molecular mechanism of circadian clock disruption in non-viral liver cancer remains unknown.

Our previous study analyzed the transcriptome of a human line of hepatoblastoma cells without viral infection (HepG2), compared with normal hepatocytes, and the gene expression data, obtained from the liver tissues of patients with hepatitis $\mathrm{C}$ virus (HCV), HCV-associated cirrhosis and liver cancer with $\mathrm{HCV}$-associated cirrhosis, using the publicly available E-MTAB-950 database (30). Despite the HepG2 cell line being revealed as a misidentified hepatocellular carcinoma cell line and subsequently identified as hepatoblastoma by the International Cell Line Authentication Committee (iclac. org/databases/cross-contaminations), the outcomes of our studies are validated because they focused on a liver cancer cell line without viral infection. Our previous analysis enabled the identification of specific clusters of genes for the various stages of liver cancer, and allowed the isolation of a network of 26 HUB genes that specifically control critical metabolic functions, independent of the viral infection (30). All 26 HUB genes were revealed to encode intrinsically disordered proteins (IDPs), thus they exhibited multifunctional behaviors and were involved in metabolic cellular control.

The present study aimed to identify whether these HUB genes (and associated proteins) were components of the network that controls the human circadian rhythm. Therefore, the network of genes involved in circadian rhythms was extracted from the human interactome to identify the nodes with high centrality and interactions with the most studied circadian gene, CLOCK. Furthermore, the association between the circadian network and 26 HUB genes in the HepG2 network was evaluated. In this way, we identified the genes linking the two main networks and evaluated if the proteins coded from them have high disorder propensities. Finally, comparing the deregulated microRNA (miRNA/miR) in HepG2 cells with miRNAs in normal hepatocytes, the target genes were predicted in order to evaluate if there were miRNAs involved in the network between HepG2 cells and circadian networks.

\section{Materials and methods}

Network analysis. A network of 71 genes was extracted, from the human interactome compiled from various databases, including Pathway Commons (31), Biological General Repository for interaction Datasets (BioGRID) (32), Human Protein Reference Database (33), ConsensusPathDB (34), Database of Interacting Proteins (35) and the Breast Cancer Information Core and Michigan Molecular Interactions (36), and identified to be involved in circadian rhythms. Only the connected component of these 71 seed networks were considered for statistical analysis using different tools, including Network-Analyzer (37), the Database for Annotation, Visualization and Integrated Discovery (DAVID) (38) and the Biological Networks Gene Ontology tool (39). Using the Cytoscape 3.5 package (www.cytoscape.org), statistical analysis was performed to evaluate the following three measures of centrality: i) The degree, which indicates the number of interactions of a particular node with other nodes in the network; ii) the betweenness, which evaluates the importance of a node in the network and how the other interactions in the network are controlled by this node (40); iii) the closeness centrality of a node, which measures the speed of information flow through this node to reachable nodes in the network and ranges from 0-1 (41). However, the power law is used to predict the HUB nodes that have functions in the network. The power law details the functional association between two quantities, where one quantity varies as a power of another; based on the power law distribution degree, a network may be defined as scale-free indicating that 'riches get richer' (42-44).

Other topological analysis, including average characteristic path length, network density, centralization and heterogeneity were evaluated using the Cytoscape 3.5. package $(41,45)$. The characteristic path length is calculated by identifying the shortest path between all pairs of nodes, adding them and dividing by the total number of pairs. This indicates the number of steps required to get from one member of the network to another $(41,45)$. The density of a network is defined as a ratio of the number of edges to the number of possible edges (46); whereas, the centralization produces rankings identify the most important nodes in a network model (47). In particular, networks with topologies resembling a star have a centralization close to 1 , whereas decentralized networks are characterized by having a centralization of $\sim 0.47$. Furthermore, the network heterogeneity, evaluated using the Cytoscape 3.5. package, reflects the tendency of a network to contain HUB nodes (48). Finally, a cluster analysis was performed, which groups similar objects to form clusters; therefore, objects in the same cluster are more similar to each other, compared with those in other clusters. In particular, the overlapping clusters were calculated on the basis of cohesiveness quality functions (49).

Disorder propensity analysis. The associated protein sequences corresponding to the HUB genes common between circadian and liver cancer networks were extracted from the UniProt database (www.uniprot.org). To assess the proportion of residues involved in intrinsic disorder, the DisProt tool (50) was used to subdivide the sequences into three major groups extracted on the basis of similar contents of disorder (10-15\%, $16-50 \%$ and $>50 \%$ ).

Target genes prediction of miRNAs. A list of miRNAs (51) that have been identified as dysregulated in HepG 2 cells, compared with human normal hepatocytes were selected. Furthermore, predictions of miRNA complementarity to $3^{\prime}$ untranslated regions (UTRs) in mRNAs were performed using three commonly used tools for target prediction: TargetScan Human 6.2 (www.targetscan.org) (52), PITA (53) and miRanda (www. microrna.org) (54). This analysis was based on identifying conserved sites that match the seed region of each miRNA, corresponding to the position between nucleotides 2 and 8in mature miRNAs. A list of putative targets for each miRNA was obtained and those predicted from $2 / 3$ tools were selected for functional annotation analysis of pathways, which was performed using the DAVID program and by selecting the more significantly enriched pathways, with a number of genes $>60$ and $\mathrm{P}<0.05$ (38). 


\section{Results and Discussion}

Human circadian network. The network of genes involved in circadian rhythms, on the basis of seed nodes, was extracted from the human interactome, and included the following genes: Aryl Hydrocarbon Receptor Nuclear Translocator Like (ARNTL), casein kinase (CSNK)1E, inter- $\alpha$-trypsin inhibitor heavy chain family member 5 (ITIH5), replication factor C subunit 3 (RFC3), WD repeat domain 41, PER1, CSNK1D, syntrophin $\beta 2$ (SNTB2), acyl-CoA thioesterase 13, chondroitin sulfate $\mathrm{N}$-acetylgalactosaminyltransferase 1, PER2, ARNTL2, PDZ domain containing ring finger 3 , growth arrest specific 2, fibronectin leucine rich transmembrane protein 1, PER3, neuronal PAS domain protein 2, low density lipoprotein receptor, zinc finger protein (ZNF) 286A, G protein-coupled receptor (GPR)116, nuclear receptor subfamily 1 group D member 1, cyclin dependent kinase (CDK) L5, splicing factor proline and glutamine rich (SFPQ), adipogenesis regulatory factor, translocase of inner mitochondrial membrane $8 \mathrm{~A}$, basic helix-loop-helix family member E (BHLHE)40, 7-dehydrocholesterol reductase, solute carrier family 39 member 14, suppressor of cytokine signaling 2 (SOCS2), GPR6, BHLHE41, histone deacetylase 4 (HDAC4), HLF PAR bZIP transcription factor (HLF), solute carrier organic anion transporter family member $4 \mathrm{~A} 1, \gamma$-secretase activating protein, BMAL1, methyl-CpG binding protein 2 (MECP2), ETS variant 5 (ETV5), Kruppel like factor 11 (KLF11), ZNF394, D-box binding PAR bZIP transcription factor, neurexin 1, TNFAIP3 interacting protein 2 (TNIP2), exocyst complex component 1 (EXOC1), extended synaptotagmin 1 (ESYT1), nuclear receptor subfamily 1 group D member 2 , SH3 and multiple Ankyrin repeat domains 3 (SHANK3), zw10 kinetochore protein (ZW10), phospholipid scramblase 1 (PLSCR1), CLOCK, solute carrier family 2 member 1 (SLC2A1), hydrocretin receptor 2, 5-methyltetrahydrofolate-homocysteine methyltransferase (MTR), transferrin receptor (TFRC), casein kinase $1-\alpha-1$, synemin, sprouty RTK signaling antagonist 4 (SPRY4), ubiquitin specific peptidase 2, glycogen synthase kinase $3 \beta$ (GSK3B), hypoxia inducible lipid droplet associated, Scmpolycomb group protein like 1, CRY1, nuclear factor interleukin 3 regulated, ATPase $\mathrm{H}+\mathrm{K}+$ transporting $\alpha$ subunit, Ras homolog family member B, CRY2, insulin induced gene 1, unc-13 homolog A (UNC13A) and apolipoprotein L domain containing 1 (APOLD1). The human circadian network consists of 2151 nodes and 75821 interactions (Table I). The circadian network was identified to be highly centralized (0.235); a higher value of centralization indicates that the network is concentrated in the center with an overall integration towards the high degree nodes. The network density of the circadian network, which describes the proportion of potential connections in a network that are actual connections, as a measure of network effectiveness, was identified to be 0.033 . In addition, the circadian network exhibited a high value of heterogeneity, which demonstrates its tendency to contain HUB nodes. The characteristic path length was identified to be 2.373 , whereas the average number of neighbors was 70.5.

The human circadian network was demonstrated to follow the small-world rule (41), as the characteristic path length is very short. The nodes that exhibited a high centrality were small ubiquitin-like modifier 2, CDK2, heat shock protein
Table I. Statistical analysis of the genes involved in the network obtained for human circadian rhythms.

\begin{tabular}{lc} 
Statistical analysis & Circadian network \\
\hline Number of nodes & 2151 \\
Number of interactions & 75821 \\
Network density & 0.033 \\
Network centralization & 0.235 \\
Characteristic path length & 2.373 \\
Network heterogeneity & 1.012 \\
Neighbor average number & 70.498
\end{tabular}

90A, p53, nuclear respiratory factor 1 and GSK3B. As CLOCK is one of the most studied circadian genes, its sub-network was extracted from the general network of circadian genes using the Cytoscape tool, which demonstrated that it contained 87 nodes with 86 direct interactions. The analysis of the present study demonstrated that CLOCK is associated with other genes including proliferating cell nuclear antigen, PER and sirtuin 1,3 and 5 .

Association between the circadian network and 26 HUBs in the HepG2 network. Four networks have previously been compared, each obtained from the differentially expressed genes in HepG2 cells and in liver tissues from patients with $\mathrm{HCV}, \mathrm{HCV}$-associated cirrhosis and liver cancer with $\mathrm{HCV}$-associated cirrhosis, using the publicly available E-MTAB-950 with the entire human interactome as the background $(30,55)$. The aim was to discriminate between liver cancer in the presence or absence of viral infection, and to identify the presence and the function of common or specific HUB nodes in the four networks. Although HepG2 cells were revealed to be misidentified as a hepatocellular carcinoma cell line instead a hepatoblastoma by International Cell Line Authentication Committee (iclac.org/databases/cross-contaminations), the results obtained using this cell line were used, as the study focused on a liver cancer cell line without viral infection. In the present study, it was evaluated whether these specific genes in HepG2 cells were components of the circadian rhythm, by identifying their presence in the circadian network. The results of the present study demonstrated that 20/26 HUB genes [CSNK2 $\alpha 1$, SH2 domain containing (SRC), ubiquitin $\mathrm{D}$, aurora kinase B (AURKB), cytoskeleton associated protein 5 (CKAP5), replication factor $\mathrm{C}$ subunit 4 , cell division cycle 20 (CDC20), stratifin, minichromosomemaintenance complex component (MCM)6, checkpoint kinase 1, centromere protein A (CENPA), HLA-B, baculoviral IAP repeat containing 5 (BIRC5), MCM3, mitotic arrest deficient 2 like 1 (MAD2L1), MCM4, ZW10 interacting kinetochore protein (ZWINT), kinesin family member (KIF)2C, inner centromere protein (INCENP) and SPC24 NDC80 kinetochore complex component (SPC24)] were demonstrated to be components of the human circadian network (Fig. 1).

The aforementioned 20 HUB genes revealed high degree values in the circadian network, ranging between 287 and 77, indicating that these genes control a large number of metabolic functions and the flow of information via the circadian network. 
Table II. Molecular properties of the 20 HUB nodes specific for HepG2 that are common to the circadian network.

\begin{tabular}{|c|c|c|c|c|c|}
\hline Gene name & Protein code & Protein name & IDR & INT & SEQ \\
\hline AURKB & Q96GD4 & Aurora kinase B & ++ & 268 & 344 \\
\hline BIRC5 & O15392 & Baculoviral IAP repeat-containingprotein 5 & +++ & 137 & 142 \\
\hline $\mathrm{CDC} 20$ & Q12834 & Cell division cycle protein 20 homolog & ++ & 595 & 499 \\
\hline CENPA & P49450 & Histone $\mathrm{H} 3$-like centromeric protein A & ++ & 129 & 140 \\
\hline CHEK1 & O14757 & Serine/threonine-protein kinase Chk1 & ++ & 161 & 476 \\
\hline CKAP5 & Q14008 & Cytoskeleton associated protein 5 & +++ & 39 & 2032 \\
\hline CSNK2A1 & P68400 & Casein kinase II subunit alpha & ++ & 599 & 391 \\
\hline HLA-B & P01889 & HLA class I histocompatibility antigen, B-7 alpha chain & ++ & 63 & 362 \\
\hline INCENP & Q9NQS7-INCE human & Inner centromere protein & +++ & 17 & 918 \\
\hline KIF2C & Q99661 & Kinesin-like protein $\mathrm{KIF} 2 \mathrm{C}$ & ++ & 82 & 725 \\
\hline MAD2L1 & Q13257 & Mitotic spindle assembly check point protein MAD2A & + & 48 & 205 \\
\hline MCM3 & P25205 & DNA replication licensing factor MCM 3 & ++ & 171 & 808 \\
\hline MCM4 & P33991 & DNA replication licensing factor MCM 4 & ++ & 152 & 863 \\
\hline MCM6 & Q14566 & DNA replication licensing factor MCM 6 & ++ & 160 & 821 \\
\hline RFC4 & P35249 & Replication factor $\mathrm{C}$ subunit 4 & + & 68 & 363 \\
\hline SFN & P31947 & 14-3-3 protein sigma & +++ & 305 & 248 \\
\hline SPC24 & Q8NBT2 & Kinetochore protein Spc24 & +++ & 31 & 197 \\
\hline $\mathrm{SRC}$ & P12931 & Proto-oncogene tyrosine-protein kinase Src & ++ & 272 & 536 \\
\hline UBD & 15205 & Ubiquitin D & +++ & 667 & 165 \\
\hline ZWINT & 95229 & Zw10- interactor & + & 55 & 277 \\
\hline
\end{tabular}

IDR, intrinsically disordered region; +, IDR <15\%; ++, IDR 15-50\%; +++, IDR >50\%; INT, number of interactions with molecular partners (determined using BioGRID); SEQ, sequence length (number of amino acids); AURKB, Aurora kinase B; BIRC5, Baculoviral IAP repeat-containing protein 5; CDC20, Cell division cycle protein 20 homolog, CENPA, histone H3-like centromeric protein A; CHEK1, serine/threonine-protein kinase Chk1; CKAP5, cytoskeleton-associated protein 5; CSNK2A1, casein kinase II subunit- $\alpha$; HLA-B, HLA class I histocompatibility antigen, B-7 $\alpha$-chain; INCENP, inner centromere protein; KIF2C, Kinesin-like protein; MAD2L1, mitotic arrest deficient 2 like 1; MCM3, minichromosome maintenance complex component 3; RFC4, replication factor C subunit 4; SFN, 14-3-3 protein- $\sigma$; SPC24, kinetochore protein Spc24; SRC, proto-oncogene tyrosine-protein kinase Src; UBD, ubiquitin D; ZWINT, Zw10 interactor.

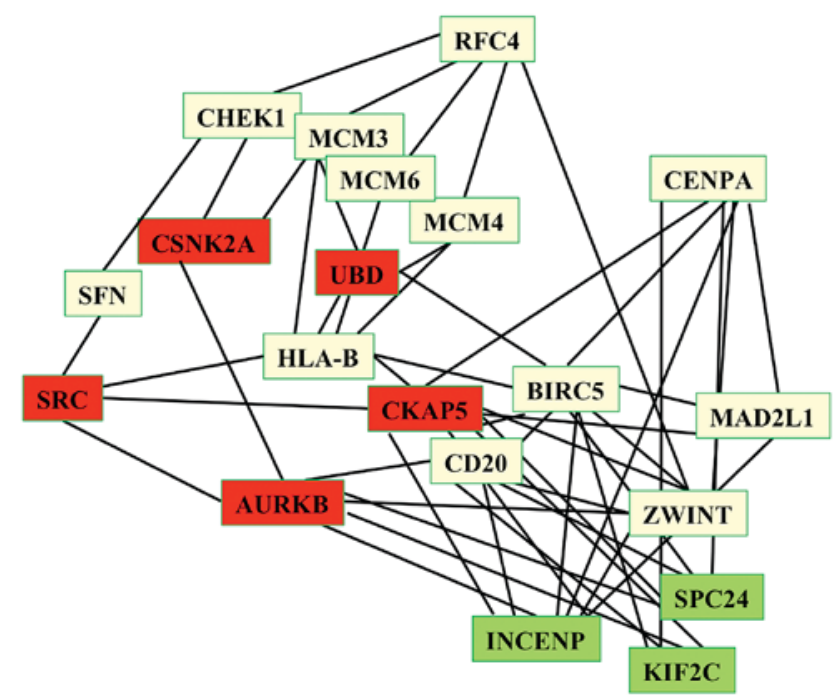

Figure 1. Schematic of the 20 HepG2 HUB genes identified to be involved in the circadian network. With respect to each other in the circadian rhythm network: Red, high degree; yellow, intermediate degree; green, low degree.

Furthermore, the results of the present study demonstrated that these 20 HUB genes interacted with 31 seed circadian genes, including CLOCK, PER1-3, CRY1-2, ARNTL2, CSNK1D, HDAC4, ZW10, CSNK1E, RFC3, MTR, SFPQ, ESYT1, transferrin receptor, GSK3B, EXOC1, SHANK3 and PLSCR1. In particular, our study has revealed that CLOCK is associated with HUB genes of the HepG2 network via CKAP5, which exhibits a high degree (217), a short path length value of 2.053 and a high value of stress centrality. Thus, CKAP5 interacts via a number of the shortest path-lengths of the network, which makes it a perfect link between circadian and HepG2 networks.

CKAP5 encodes a cytoskeleton-associated protein belonging to the TOG/XMAP215 family, and CKAP is also known as a colonic and hepatic tumor overexpressed gene protein $(56,57)$. Its coded protein serves two distinct functions in spindle formation and in the protection of kinetochore microtubules, via the control of the de-polymerization process and centrosomal microtubule assembly $(58,59)$. These two processes regulate the mitotic cell cycle via spindle formation (60) and the interaction between microtubules and the cell cortex for the directional cell movement. Notably, the results of the present study identified that CKAP5 is associated with three circadian genes (CSNK1E, CSNK1D and HDAC4) and with 10 HepG2 genes (SRC, ZWINT, AURKB, CDC20, CENPA, INCENP, MAD2L1, BIRC5, SPC24 and 
A

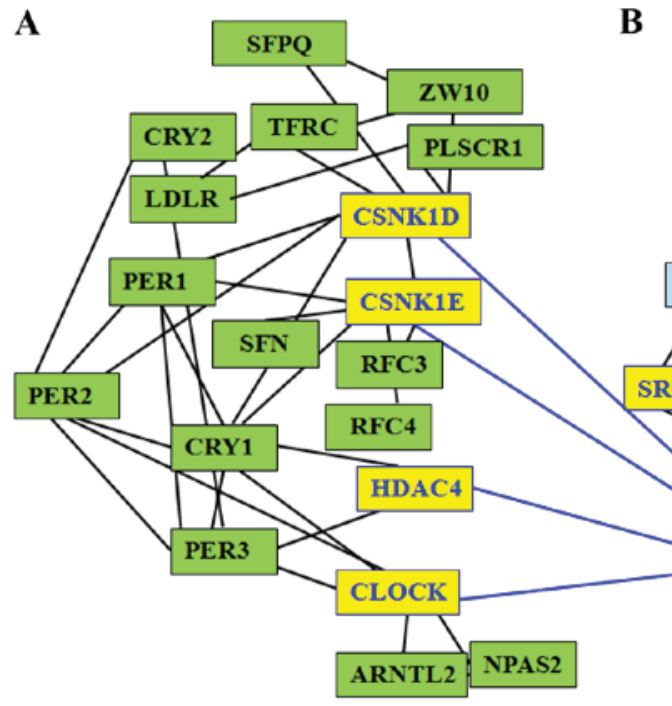

B

Figure 2. Association between (A) the circadian sub-network and (B) HepG2 HUB-sub-network. Yellow represents CKAP5, the HepG2 HUB-genes and four circadian seed genes (CLOCK, CSNK1E, CSNK1D and HDAC4), which are directly connected via CKAP5. The other genes present in circadian and HepG2 HUB sub-networks are indicated in green and blue, respectively. CKAP5, cytoskeleton associated protein 5; CLOCK, clock circadian regulator; CSNK, casein kinase; HDAC4, histone deacetylase 4.

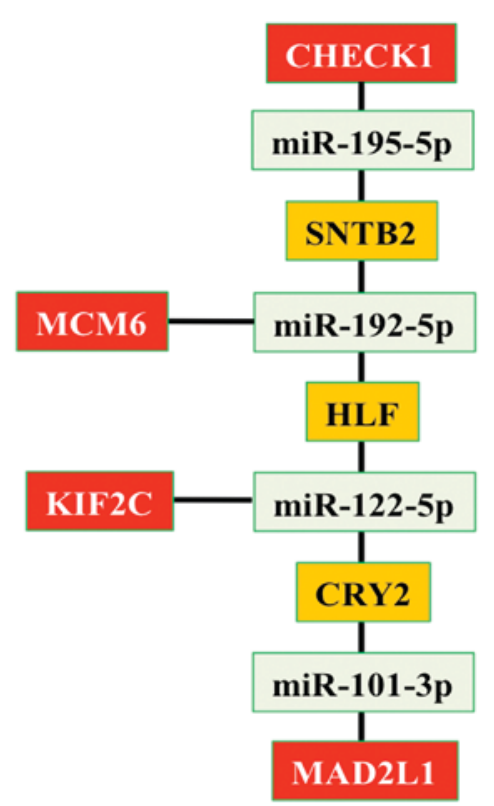

Figure 3. Associations between genes and miRNAs that link HepG2 and circadian networks. In details, four genes (CHECK1, KIF2C, MCM6 and MAD2L1) are reported in red; four miRNAs (miR-195-5p, miR-192-5p, miR-122-5p and miR-101-3p) are reported in green and three genes (SNTB2, HLF and CRY2) that correlate the miRNAs are reported in orange. miR, microRNA; SNTB2, syntrophin $\beta 2$; MCM6, minichromosome maintenance complex component 6; HLF, HLF PAR bZIP transcription factor KIF2C, kinesin family member 2C; CRY2, cryptochrome circadian clock 2; MAD2L1, mitotic arrest deficient 2 like 1; CHECK1, checkpoint kinase 1.

KIF2C; Fig. 2). Therefore, the two sub-networks of circadian genes and HepG2 genes connected via CKAP5 may be disturbed by any alteration associated with circadian and liver cancer genes. Due to the close associations between nodes, a putative perturbing stressor, for example an alteration of a circadian gene, can induce a perturbation to the global network, and thus, cancer progression (61).
Structural analysis on common nodes between HepG2 and circadian networks. As all HUB genes identified in our network analysis encode for proteins, it is crucial to understand whether the genes possess specific structural features. Previously, it was demonstrated that the metabolic sub-network specific for liver cancer is formed only by IDPs $(30,55)$. Structural flexibility and binding plasticity enable IDPs to interact with a broad range of molecular partners $(30,55)$. Therefore, in the present study, disorder propensity and the number of molecular partners, with which these proteins can interact, was evaluated using DisProt and BioGRID tools, respectively. As presented in Table II, the proteins encoded by the genes common to the circadian and HepG2 networks belonged to the IDP family. In particular, 3, 11 and 7 proteins exhibited 15\%, 16-50\% and $>50 \%$ ID regions (IDRs), respectively. Subsequently, the physical interactions between all proteins encoded by the genes common to the circadian and HepG2 network and other proteins with which they can interact were analyzed, and demonstrated that they are able to form between 17 and 667 interactions. This result supports our hypothesis that the flexibility of the disordered regions functions in the establishment a high number of interactions.

Association between miRNAs and genes of the human circadian and HepG2 networks. Following the identification of the associations between circadian and HepG2 networks, and demonstrating that genes common to the two networks encode IDPs, the presence of miRNAs involved in the sub-network between HepG2 and circadian genes was evaluated. A prior study identified the deregulated miRNAs in HepG2 cells compared with normal hepatocytes (51). A total of 11 downregulated (miR-146b-5p, miR-195, miR-122, miR-122a, miR-375, miR-885-5p, miR-768-5p, miR-101, miR-192, miR-194 and miR-215) and 2 upregulated miRNAs (miR-221 and miR-99b) were identified (51). Therefore, in the present study, the target genes of these 13 miRNAs were 
predicted using the three aforementioned tools. The results of the present study identified 5415 target genes belonging to different metabolic pathways including axon guidance, hippo signaling, endocytosis, phosphoinositide 3-kinase/protein kinase B (AKT) signaling, RAS signaling, RAP1 signaling and chemokine signaling.

To determine the target genes involved in the HepG2 and circadian networks, genes common to the identified 5,415 target genes and the circadian genes were identified. The results of the present study identified 28 genes (APOLD1, BHLHE40, BHLHE41, CLOCK, CRY1, CRY2, extended synaptotagmin 1, ETV5, HDAC4, HLF, ITIH5, KLF11, MECP2, MTR, nuclear receptor subfamily 2 group D member 2, PER1, PER2, PER3, RFC3, SLC2A1, SNTB2, SOCS2, SPRY4, TFRC, TNIP2, UNC13a and ZW10) that are common between circadian genes and the 5415 identified target genes. In addition, four genes (checkpoint kinase 1, KIF2C, MCM6 and MAD2L1), that associated the HepG2 network with the circadian network, were targeted by the following four miRNAs: miR-195-5p, miR-192-5p, miR-122-5p and miR-101-3p. The present study identified three genes (SNTB2, HLF and CRY2) that correlated four miRNAs (miR-195-5p, miR-192-5p, miR-122-5p and miR-101-3p) between them (Fig. 3).

Previous studies have suggested that liver cancer is associated with abnormalities in circadian rhythms $(26,62)$ due to alterations in the expression of certain circadian genes in cancerous cells, induced by hypoxia (24), or the overexpression of the mammalian timeless protein (23), a protein that controls chromosome integrity, growth and development. The present study was, to the best of our knowledge, the first to identify a sub-set of HUB genes consisting of genes present in the HepG2 cell network, and involved in cancer progression, in common with human circadian rhythm genes. The results of the present study revealed the following: i) CLOCK is associated, via CKAP5, with the HUB genes of the HepG2 network; ii) CKAP5 is associated with three other circadian genes (CSNK1E, CSNK1D and HDAC4), and with 10HepG2 genes (SRC, ZWINT, AURKB, CDC20, CENPA, INCENP, MAD2L1, BIRC5, SPC24 and KIF2C); iii) the genes linking the circadian system and liver cancer codify for proteins that exhibit IDRs; iv) a sub-panel of seven genes and three miRNAs link human circadian rhythms with liver cancer in a single network.

Masri et al (63) demonstrated that the liver operates as an exclusive endogenous metabolic reorganizer in tumor-bearing mice (63). Notably, associations between cancer and circadian genes are maintained while the pro-inflammatory response of the liver is altered and leads to the disturbance of AKT, AMP-activated protein kinase and sterol regulatory element binding protein signaling, which, in turn, affects glucose and lipid metabolism. These results demonstrate the requirement to study associations between the circadian rhythms in liver cancer and/or other types of cancer (63). In this context, the results of the present study indicated that further studies are required to determine how the structural perturbation of the HUB nodes in liver cancer may trigger significant and widespread sources of functional changes, which consequently may produce the distinct metabolic functions of cancer cells.

\section{References}

1. Czeisler CA and Klerman EB: Circadian and sleep-dependent regulation of hormone release in humans. Recent ProgHorm Res 54: 97-130, 1999.

2. Dibner $\mathrm{C}$ and Schibler U: Circadian timing of metabolism in animal models and humans. J Intern Med 277: 513-527, 2015.

3. Harfmann BD, Schroder EA and Esser KA: Circadian rhythms the molecular clock and skeletal muscle. J Biol Rhythms 30: 84-94, 2015.

4. West AC and Bechtold DA: The cost of circadian desynchrony: Evidence insights and open questions. BioEssays 37: 777-788, 2015.

5. Fares S, Hermens DF, Naismith SL, White D, Hickie IB and Robillard R: Clinical correlates of chronotypes in young persons with mental disorders. Chronobiol Int 32: 1183-1191, 2015.

6. Geoffroy PA, Etain B, Sportiche S and Bellivier F: Circadian biomarkers in patients with bipolar disorder: Promising putative predictors of lithium response. Int J Bipolar Disord 2: 28, 2014.

7. Geoffroy PA, Etain B, Franchi JA, Bellivier F and Ritter P: Melatonin and melatonin agonists as adjunctive treatments in bipolar disorders. Curr Pharm Des 21: 3352-3358, 2015.

8. Benedetti F, Riccaboni R, Dallaspezia S, Locatelli C, Smeraldi E and Colombo C: Effects of CLOCK gene variants and early stress on hopelessness and suicide in bipolar depression. Chronobiol Int 32: 1156-1161, 2015.

9. Russo M, Mahon K, Shanahan M, Ramjas E, Solon C, Purcell SM and Burdick KE: The relationship between sleep quality and neurocognition in bipolar disorder. J Affect Disord 187: 156-162, 2015.

10. Challet E: Keeping circadian time with hormones. Diabetes Obes Metab 17: 76-83, 2015.

11. Anisimov VN, Vinogradova IA, Panchenko AV, Popovich IG and Zabezhinski MA: Light-at-night-induced circadian disruption cancer and aging. Curr Aging Sci 5: 170-177, 2012.

12. Belancio VP: LINE-1 activity as molecular basis for genomic instability associated with light exposure at night. Mob Genet Elements 5: 1-5, 2015.

13. Savvidis C and Koutsilieris M: Circadian rhythm disruption in cancer biology. Mol Med 18: 1249-1260, 2012.

14. Uth K and Sleigh R: Deregulation of the circadian clock constitutes a significant factor in tumorigenesis: A clockwork cancer. Part II In vivo studies. Biotechnol Biotechnol Equip 28: 379-386, 2014.

15. Michael AK, Harvey SL, Sammons PJ, Anderson AP, Kopalle HM, Banham AH and Partch CL: Cancer/Testis antigen PASD1 silences the circadian clock. Mol Cell 58: 743-754, 2015.

16. Kiss Z and Ghosh PM: Woman in cancer thematic review: Circadian rhythmicity and the influence of 'clock' genes on prostate cancer. Endocr Relat Cancer 23: T123-T134, 2016.

17. Gutiérrez-Monreal MA, Villela L, Baltazar S, Perfecto-Avalos Y, Cardineau GA and Scott SP: A PER3 polymorphism is associated with better overall survival in diffuse large B-cell lymphoma in Mexican population. Cancer Biomark 15: 699-705, 2015.

18. Li J, Chen R, Ji M, Zou SL and Zhu LN: Cisplatin-based chronotherapy for advanced non-small cell lung cancer patients: A randomized controlled study and its pharmacokinetworkics analysis. Cancer Chemother Pharmacol 76: 651-655, 2015.

19. Mitchell MI and Engelbrecht AM: Circadian rhythms and breast cancer: The role of Per2 in doxorubicin-induced cell death. J Toxicol 2015: 392360, 2015.

20. Poole EM, Schernhammer E, Mills L, Hankinson SE and Tworoger SS: Urinary melatonin and risk of ovarian cancer. Cancer Causes Control 26: 1501-1506, 2015.

21. Schmutz I, Albrecht U and Ripperger JA: The role of clock genes and rhythmicity in the liver. Mol Cell Endocrinol 349: 38-44, 2012.

22. Vollmers C, Gill S, DiTacchio L, Pulivarthy SR, Le HD and Panda S: Time of feeding and the intrinsic circadian clock drive rhythms in hepatic gene expression. ProcNatl Acad Sci USA 106: 21453-21458, 2009.

23. Elgohary N, Pellegrino R, Neumann O, Elzawahry HM, Saber MM, Zeeneldin AA, Geffers R, Ehemann V, Schemmer P, Schirmacher $\mathrm{P}$ and Longerich T: Protumorigenic role of timeless in hepatocellular carcinoma. Int J Oncol 46: 597-606, 2015.

24. Yu C, Yang SL, Fang X, Jiang JX, Sun CY and Huang T: Hypoxia disrupts the expression levels of circadian rhythm genes in hepatocellular carcinoma. Mol Med Rep 11: 4002-4008, 2015.

25. Li H, Lu YF, Chen H and Liu J: Dysregulation of metallothionein and circadian genes in human hepatocellular carcinoma. Chronobiol Int 20: 192-202, 2016. 
26. Yang SL, Yu C, Jiang JX, Liu LP, Fang X and Wu C: Hepatitis B virus $\mathrm{X}$ protein disrupts the balance of the expression of circadian rhythm genes in hepatocellular carcinoma. Oncol Lett 8 : 2715-2720, 2014

27. Turati F, Talamini R, Pelucchi C, Polesel J, Franceschi S, Crispo A, Izzo F, La Vecchia C, Boffetta P and Montella M: Metabolic syndrome and hepatocellular carcinoma risk. Br J Cancer 108: 222-658, 2013.

28. Polesel J, Zucchetto A, Montella M, Dal Maso L, Crispo A, La Vecchia C, Serraino D, Franceschi S and Talamini R: The impact of obesity and diabetes mellitus on the risk of hepatocellular carcinoma. Ann Oncol 20: 353-357, 2009.

29. Shivappa N, Hébert JR, Polesel J, Zucchetto A, Crispo A Montella M, Franceschi S, Rossi M, La Vecchia C and Serraino D Inflammatory potential of diet and risk for hepatocellular cancer in a case-control study from Italy. Br J Nutr 115: 324-331, 2016.

30. Singh S, Colonna G, Di Bernardo G, Bergantino F, Cammarota M, Castello $\mathrm{G}$ and Costantini S: The gene expression profiling of hepatocellular carcinoma by a network analysis approach shows a dominance of intrinsically disordered proteins (IDPs) between hub nodes. Mol Biosyst 11: 2933-2945, 2015.

31. Cerami EG, Gross BE, Demir E, Rodchenkov I, Babur O, Anwar N, Schultz N, Bader GD and Sander C: Pathway commons a web resource for biological pathway data. Nucleic Acids Res 39 (Database issue): D685-D690, 2011.

32. Stark C, Breitkreutz BJ, Reguly T, Boucher L, Breitkreutz A and Tyers M: BioGRID: A general repository for interaction datasets. Nucleic Acids Res 34 (Database issue): D535-D539, 2006.

33. Peri S, Navarro JD, Amanchy R, Kristiansen TZ Jonnalagadda CK, Surendranath V, Niranjan V, Muthusamy B, Gandhi TK, Gronborg M, et al: Development of human protein reference database as an initial platform for approaching systems biology in humans. Genome Res 13: 2363-2371, 2003.

34. Kamburov A, Wierling $C$, Lehrach $H$ and Herwig $R$ : ConsensusPathDB--a database for integrating human functional interaction networks. Nucleic Acids Res 37 (Database issue): D623-D628, 2009.

35. Xenarios I, Salwinski L, Duan XJ, Higney P, Kim SM and Eisenberg D: DIP, the database of interacting proteins: A research tool for studying cellular networks of protein interactions. Nucleic Acids Res 30: 303-305, 2012

36. Jayapandian M, Chapman A, Tarcea VG, Yu C, Elkiss A, Ianni A Liu B, Nandi A, Santos C, Andrews P, et al: Michigan Molecular Interactions (MiMI): Putting the jigsaw puzzle together. Nucleic Acids Res 35 (Database issue): D566-D571, 2007.

37. Cline MS, Smoot M, Cerami E, Kuchinsky A, Landys N, Workman C, Christmas R, Avila-Campilo I, Creech M, Gross B, et al: Integration of biological networks and gene expression data using Cytoscape. Nat Protoc 2: 2366-2382, 2007.

38. Huang da W, Sherman BT and Lempicki RA: Systematic and integrative analysis of large gene lists using DAVID bioinformatics resources. Nat Protoc 4: 44-57, 2009.

39. Maere S, Heymans K and Kuiper M: BiNGO: A cytoscape plugin to assess overrepresentation of gene ontology categories in biological networks. Bioinformatics 21: 3448-3469, 2005.

40. Yoon J, Blumer A and Lee K: An algorithm for modularity analysis of directed and weighted biological networks based on edge-betweenness centrality. Bioinformatics 22: 3106-3108, 2006.

41. Newman MEJ: A measure of betweenness centrality based on random walks. Soc Networks 27: 39-54, 2005.

42. Barabási AL, Gulbahce N and Loscalzo J: Network medicine: A network-based approach to human disease. Nat Rev Genet 12 56-68, 2011

43. Sharma A, Costantini S and Colonna G: The protein-protein interaction network of the human Sirtuin family. Biochim Biophys Acta 1834: 1998-2009, 2013.

44. Wu J, Tan Y, Deng H and Zhu D: Relationship between degree-rank function and degree distribution of protein-protein interaction networks. Comput Biol Chem 32: 1-4, 2008.
45. Wu J, Tan YJ, Deng HZ and Zhu DZ: A new measure of heterogeneity of complex networks based on degree sequence. Unifying Themes in Complex Systems 66-73, 2010.

46. Dong $\mathrm{J}$ and Horvath S: Understanding network concepts in modules. BMC Syst Biol 1: 24, 2007.

47. Freeman LC: Centrality in social networks conceptual clarification. Soc Networks 1: 215-239, 1978.

48. Estrada E: Quantifying network heterogeneity. Phys Rev E Stat Nonlin Soft Matter Phys 82: 066102, 2010.

49. Nepusz T, Yu H and Paccanaro A: Detecting overlapping protein complexes in protein-protein interaction networks. Nat Methods 9: 471-472, 2012.

50. Peng K, Vucetic S, Radivojac P, Brown CJ, Dunker AK and Obradovic Z: Optimizing long intrinsic disorder predictors with protein evolutionary information. J Bioinform Comput Biol 3: 35-60, 2015.

51. He XX, Chang Y, Meng FY, Wang MY, Xie QH, Tang F, Li PY, Song YH and Lin JS: MicroRNA-375 targets AEG-1 in hepatocellular carcinoma and suppresses liver cancer cell growth in vitro and in vivo. Oncogene 31: 3357-3369, 2012

52. Lewis BP, Burge CB and Bartel DP: Conserved seed pairing, often flanked by adenosines indicates that thousands of human genes are microRNA targets. Cell 120: 15-20, 2005.

53. Kertesz M, Iovino N, Unnerstall U, Gaul U and Segal E: The role of site accessibility in microRNA target recognition. Nat Genet 39: 1278-1284, 2007.

54. John B, Enright AJ, Aravin A, Tuschl T, Sander C and Marks DS: Human MicroRNA targets. PLoS Biol 3: e264, 2005.

55. Costantini S, Di Bernardo G, Cammarota M, Castello G and Colonna G: Gene expression signature of human HepG2 cell line. Gene 518: 335-345, 2013.

56. Nagase T, Miyajima N, Tanaka A, Sazuka T, Seki N, Sato S, Tabata S, Ishikawa K, Kawarabayasi Y,Kotani H, et al: Prediction of the coding sequences of unidentified human genes. III. The coding sequences of 40 new genes (KIAA0081-KIAA0120) deduced by analysis of cDNA clones from human cell line KG-1. DNA Res 2: 37-43, 1995.

57. Charrasse S, Mazel M, Taviaux S, Berta P, Chow T and Larroque C: Characterization of the cDNA and pattern of expression of a new gene over-expressed in human hepatomas and colonic tumors. Eur J Biochem 234: 406-413, 1995.

58. Takeshita N, Mania D, Herrero S, Ishitsuka Y, Nienhaus GU, Podolski M, Howard J and Fischer R: The cell-end marker TeaA and the microtubule polymerase AlpA contribute to microtubule guidance at the hyphal tip cortex of Aspergillus nidulans to provide polarity maintenance. J Cell Sci 126: 5400-5411, 2013

59. Wu Z, Chen Y, Yang T, Gao Q, Yuan M and Ma L: Targeted ubiquitination and degradation of G-protein-coupled receptor kinase 5 by the DDB1-CUL4 ubiquitin ligase complex. PLoS One 7: e43997, 2012.

60. Golsteyn RM, Mundt KE, Fry AM and Nigg EA: Cell cycle regulation of the activity and subcellular localization of Plk1, a human protein kinase implicated in mitotic spindle function. J Cell Biol 129: 1617-1628, 1995 .

61. Li JZ, Bunney BG, Meng F, Hagenauer MH, Walsh DM, Vawter MP, Evans SJ, Choudary PV, Cartagena P, Barchas JD, et al: Circadian patterns of gene expression in the human brain and disruption in major depressive disorder. Proc Natl Acad Sci USA: 110: 9950-9955, 2013.

62. Vinciguerra M, Mazzoccoli G, Piccoli C, Tataranni T, Andriulli A and Pazienza V: Exploitation of host clock gene machinery by hepatitis viruses B and C. World J Gastroenterol 19: 8902-8909, 2013.

63. Masri S, Papagiannakopoulos T, Kinouchi K, Liu Y, Cervantes M, Baldi P, Jacks T and Sassone-Corsi P: Lung adenocarcinoma distally rewires hepatic circadian homeostasis. Cell 165: 896-909, 2016. 